\title{
Prótesis en T de Montgomery como parte del tratamiento inicial de la fistula traqueoesofágica no tumoral en pacientes con mal estado nutricional
}

\author{
Montgomery T-prosthesis as part of the initial treatment \\ of non-tumoral tracheoesophageal fistula in patients \\ with poor nutritional status
}

\begin{abstract}
Álvaro Ortiz Naretto',,2, Glenda Ernst², Martín Korsunsky', Alejandro Sansostera', Alejandro Briosso', Bibiana Vazquez', Sebastián Gando', Roberto Duré'

\section{RESUMEN}

La fístula traqueoesofágica (FTE) es una rara complicación que se produce en pacientes intubados. Esta genera una comunicación entre esófago y tráquea que podría estar relacionada con el aumento de infecciones respiratorias, entre otras causas. Su resolución es quirúrgica y ha sido demostrado que el estado nutricional previo de los pacientes está asociado con el éxito de la cirugía. En este estudio se incluyeron pacientes traqueotomizados por asistencia ventilatoria mecánica (AVM) prolongada con diagnóstico de FTE, a los cuales se les colocó prótesis traqueales en T (Montgomery) para mejorar la alimentación vía oral. Esto contribuyó a mejorar el estado nutricional y por consiguiente la posibilidad de ser candidatos a cirugía para sellar la fístula.

Se colocó prótesis en T a 9 pacientes que requirieron $9.5 \pm 1.5$ meses en promedio para mostrar una mejoría de las proteínas a valores normales. Durante este período no presentaron complicaciones asociadas a FTE, ni infecciones respiratorias. El tratamiento quirúrgico fue exitoso en todos los casos. Se demostró a partir de esta serie de pacientes que el manejo endoscópico resultó efectivo para mejorar las condiciones nutricionales de los pacientes y alcanzar el éxito de la cirugía. Nuevos estudios con mayor cantidad de pacientes son necesarios para confirmar estos hallazgos. La colocación de una prótesis traqueal en T Montgomery podría permitir la alimentación completa vía oral del paciente, como una alternativa segura y eficaz.

Palabras clave: fístula traqueoesofágica; broncoscopia; prótesis tipo $\top$ toráci-

ABSTRACT

Tracheoesophageal fistula (TEF) is a rare complication that occurs in intubated patients. This generates a communication between esophagus and trachea, is related to the increase of respiratory infections. Its resolution is surgical, and it has been demonstrated that patients' previous nutritional status is related to surgical success.

This study included patients tracheotomized due to prolonged assisted mechanical ventilation (AVM) with a diagnosis of TEF, and who received a Montgomery T-tube tracheal prostheses to improve oral intake. This contributed to improve their nutritional status and therefore enabling them to be candidates for surgery to seal the fistula.

The Montgomery T-tube prosthesis was placed on nine patients, who required $9.5 \pm 1.5$ months on average to show an improvement of their protein level to normal values. During this period, there were no complications associated with FTE and no respiratory infections developed. Surgical treatment was successful in all cases.

It was demonstrated from this series of patients that the endoscopic management was effective to improve the nutritional conditions of the patients, and to achieve surgical success. New studies with a larger number of patients are necessary to confirm these findings. The placement of a Montgomery T-tube tracheal prosthesis in these patients allowed complete oral feeding as being a safe and effective alternative.
\end{abstract} ca; prótesis de Montgomery.
Keywords: esophageal tracheal fistula; bronchoscopy; thoracic T-type prosthesis; Montgomery prosthesis.
I. Unidad de Endoscopia Peroral, Hospital "Francisco Javier

Muñiz", Ciudad Autónoma de Buenos Aires.

2. Servicio de Docencia, Hospital Británico de Buenos Aires.

Correspondencia: Dr. Álvaro Ortiz Naretto. Hospital "Francisco Javier Muñiz". Uspallata 2272, CI282AEN CABA. Email: aortiz007@gmail.com

Los autores declaran no tener conflictos de intereses.

Recibido: 05/06/2018 |Aceptado: 13/07/2018

\section{INTRODUCCIÓN}

La fístula traqueoesofágica (FTE) benigna constituye una severa complicación que afecta a 0.5 de los pacientes con tubo orotraqueal y se caracteriza por comunicar patológicamente el esófago y la tráquea ${ }^{1-3}$. Se debe sospechar en pacientes que presentan tos durante la deglución, neumonías recurrentes y en aquellos bajo asistencia ventilatoria mecánica (AVM) que presentan dificultades en la ventilación, distensión abdominal, salida del contenido gástrico por el tubo orotraqueal (TOT) o de la traqueostomía ${ }^{1}$. Su desa- 
rrollo podría estar relacionado con la presión ejercida por el balón de sellado traqueal ${ }^{2}$.

El estado nutricional de los pacientes con FTE en la Terapia Intensiva (TI) se ve comprometido ya que estas lesiones permiten el paso de contenido esofágico tanto a la tráquea como a las vías respiratorias distales, sepsis y dificultad en la nutrición ${ }^{4}$.

La cirugía constituye actualmente el tratamiento de elección de la FTE ya que posibilita el cierre del orificio de la fístula ${ }^{5}$. Sin embargo, el momento de realización de la cirugía en estos pacientes con mal estado nutricional podría estar asociado al éxito o fracaso del procedimiento.

Han sido propuestos diferentes enfoques para el manejo de FTE; la principal estrategia de manejo es la resolución quirúrgica inmediata luego del diagnóstico ${ }^{1,6-8 .}$ Sin embargo, se han descripto complicaciones tales como, mortalidad entre 2.6 y el $10 \%$ y refistulización ocasional ${ }^{4,5,7}$. Dichas complicaciones posquirúrgicas, que se incrementan cuando están asociadas a un estado nutricional inadecuado de los pacientes, podrían cuestionar su realización.

Este estudio propuso el cierre de la comunicación traqueoesofágica por medio de una prótesis traqueal de Montgomery, permitiendo la alimentación vía oral para favorecer una mejoría del estado nutricional de los pacientes al permitir la alimentación por vía oral y evitar las complicaciones asociadas a las FTE tales como la neumonía aspirativa. Más aún; los pacientes lograron una alimentación completa aun fuera del ámbito hospitalario. De esta manera, se pudieron alcanzar las condiciones quirúrgicas adecuadas, disminuyendo los riesgos de complicaciones pre- y posoperatorias. Esta decisión resultó innovadora ya que el tubo en T de Montgomery se describe en la literatura como herramienta en el posoperatorio para estabilidad de la cirugía, no como herramienta para estabilizar el estado crítico nutricional del paciente ${ }^{4}$.

El objetivo del presente trabajo fue describir la experiencia de colocar una prótesis traqueal en $T$ (Montgomery) para mejorar la alimentación vía oral y la consecuente mejoría del estado nutricional (objetivado por valores en sangre de proteínas totales y albúmina) en pacientes traqueotomizados por AVM prolongada con diagnóstico de FTE que no eran candidatos a cirugía al momento del diagnóstico por su mal estado clínico nutricional.

\section{MATERIALESY MÉTODOS}

\section{Diseño}

Se realizó un estudio de prospectivo, descriptivo de todos los pacientes ingresados con diagnóstico y/o sospecha de FTE entre los años 2002 a 2009 en un centro de referencia endoscópica. Todos los pacientes avalaron la colocación de la prótesis voluntariamente y firmaron

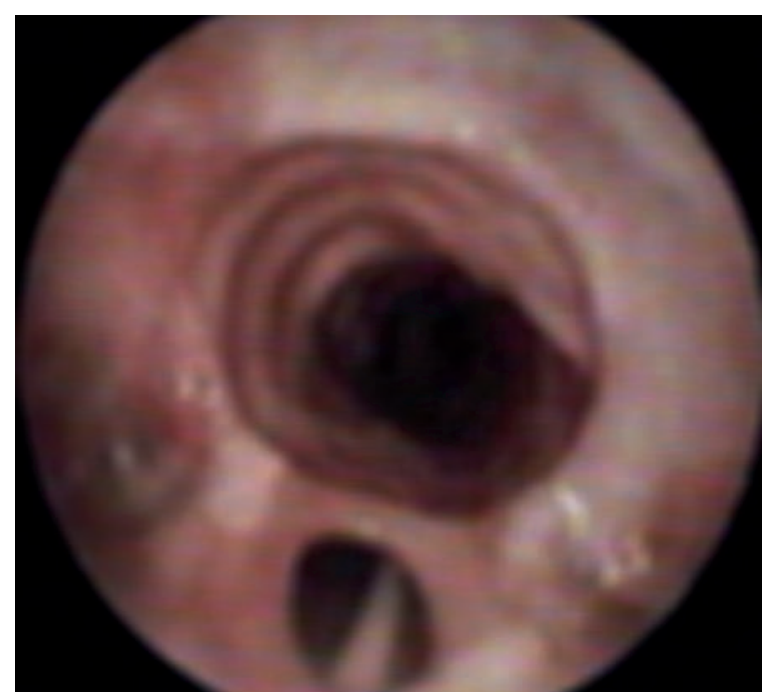

Figura I. Se observa en el tercio superior de la tráquea el orificio de la fistula de bordes netos y epitelizada en la pars membranosa. Se observa la sonda de alimentación enteral.

un consentimiento informado autorizando la realización de las prácticas endoscópicas.

\section{Población}

Se evaluaron todos los pacientes con sospecha de FTE mediante una fibrobroncoscopia (FBC) como método diagnóstico. Solo fueron sometidos a la colocación de la prótesis aquellos pacientes cuyo estado nutricional (proteínas totales/albúmina) fue anormal y/o se encontraban emaciados, por lo que la cirugía al momento del diagnóstico estaba contraindicada. Se definió estado nutricional anormal por parámetros de laboratorio: valores de proteínas totales en sangre $\leq 6.5 \mathrm{~g} / \mathrm{dl}$ y de albúmina $\leq 3.3 \mathrm{~g} / \mathrm{dl}$. Se evaluaron los valores de proteínas totales/albúmina en sangre de los pacientes al momento del diagnóstico y al momento de la mejoría clínica para definir la indicación quirúrgica. Alcanzado el estado nutricional adecuado y con la mejoría de los valores de proteínas totales/albúmina en sangre, se retiró la prótesis tipo T, se evaluó la persistencia de FTE y la necesidad indicación quirúrgica. Se realizó un seguimiento de un año posterior al retiro de la prótesis.

\section{Procedimientos endoscópicos}

Se utilizó la prótesis tipo T torácica (Stening y Cía.) para ocluir el orificio fistuloso. Las prótesis se seleccionaron en cada paciente de manera que evitaran la filtración desde el esófago durante la deglución. Todos los procedimientos se realizaron bajo neuroleptoanalgesia utilizando un fibrobroncoscopio Pentax FB 15B y Olympus FB 18F, y broncoscopio rígido tipo Chevallier Jackson Gil López y Cía. números 8 y 9. Se evaluaron los trastornos de la deglución por parámetros clínicos y $\mathrm{FEES}^{9}$ (fiberoptic endoscopic evaluation of swallowing). La seriada esofágica solo se realizó si había necesidad de diferenciar broncoaspiración translaríngea o por la fístula. 


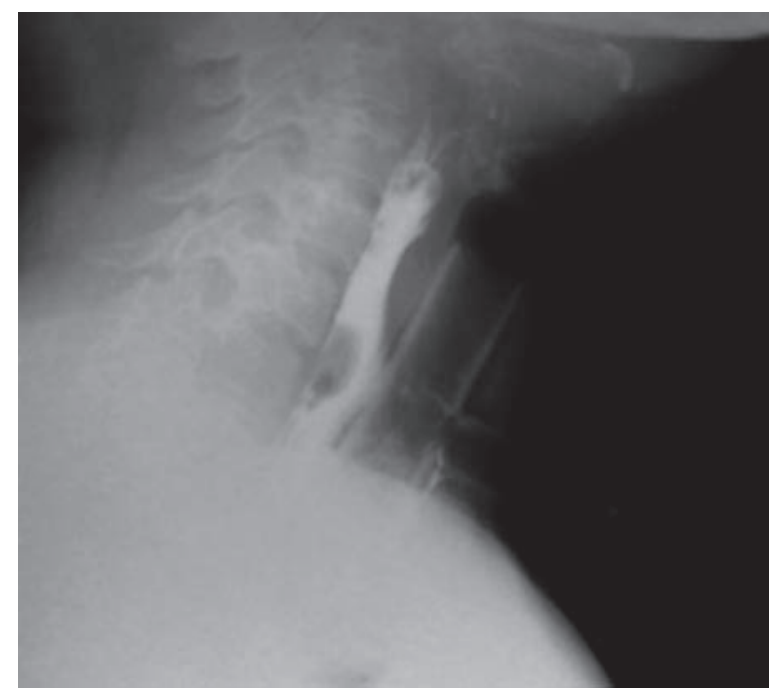

Figura 2. Seriada esofágica que muestra la persistencia del orificio de la fistula pero sin pasaje del contraste a la vía respiratoria al año de colocada la prótesis tipo $T$ de tórax que ocluye el orificio fistuloso.

\section{Análisis estadístico}

Se realizó un análisis de estadística descriptiva sobre la población y se compararon variables continuas de distribución normal apareadas con test de T. Los resultados se muestran como promedio y desvío estándar.

\section{RESULTADOS}

\section{Características de la población estudiada}

Durante el período evaluado ( 7 años) se diagnosticaron 10 pacientes con FTE que fueron derivados al Servicio de Endoscopia para su resolución definitiva. El 66.7\% eran varones con una edad promedio de 41.4 años (1666 años). Sus características se detallan en la Tabla 1. La causa de la FTE fue en nueve pacientes por AVM prolongado y solo en uno secundaria a complicación por cuerpo extraño en el esófago. Los nueve pacientes con FTE por AVM prolongada estuvieron ventilados en promedio 22.7 días (rango: 3-45 días). En un paciente se había intentado el cierre quirúrgico de la FTE, el cual fracasó, con persistencia de la fístula en el posoperatorio, y luego fue derivado a nuestro servicio. Se excluyó para colocación de prótesis un paciente porque presentaba buen estado nutricional, con niveles de proteínas totales y albúmina normales, por lo que fue derivado a cirugía para su resolución quirúrgica inmediata.

\section{Diagnóstico de la FTE}

En cinco de los pacientes estudiados se sospechó la presencia de FTE durante la AVM por la salida de alimento periostoma y/o dificultad para ventilarlos en forma adecuada, y en cuatro pacientes la sospecha de la FTE fue luego de su salida de la unidad de TI incluyendo en este último grupo el paciente que fue derivado a cirugía sin espera. Finalmente un paciente se diagnosticó a la semana luego de la extracción de cuerpo extraño en esófago (prótesis dental).
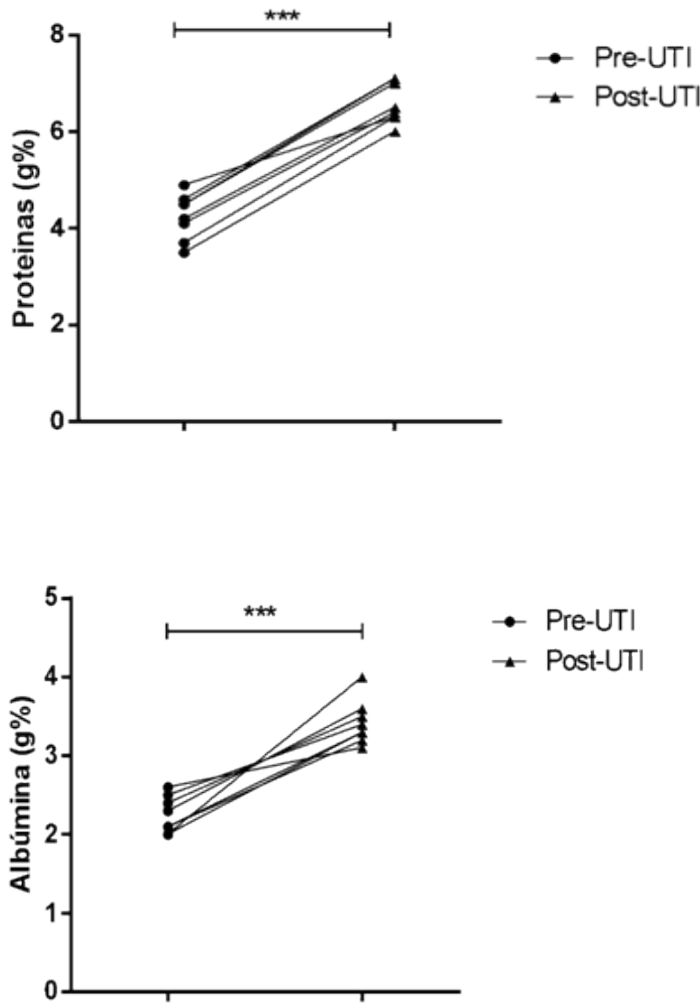

Figura 3. A) Modificación de la cantidad de proteínas al momento del diagnóstico y posteriores a la colocación del tubo en T o prequirúrgico. B) De manera similar al punto A), se muestra la modificación de los valores de la albúmina.

La confirmación de la FTE se realizó siempre por FBC. En caso de estar el paciente en AVM se retiró el tubo orotraqueal o cánula de traqueotomía para poder visualizar la fístula (Figura 1). La fístula se ubicó entre tercio medio y superior de la tráquea en la totalidad de los pacientes, con un tamaño promedio del orificio fistuloso al momento del diagnóstico de $1.29 \mathrm{~cm}$ (rango: 0.3-2.3).

Se realizó control clínico de los pacientes evaluando la presencia de tos o neumonía, y se descartó trastornos de la deglución por FEES en los 9 pacientes. Solo en un paciente se realizó una seriada esofágica por la necesidad de diferenciar broncoaspiración translaríngea o por la fístula (Figura 2).

\section{Colocación del Montgomery}

Los nueve pacientes que habían sido rechazados de cirugía de forma inmediata por su mal estado nutricional, que estaban traqueostomizados - uno era fracaso de cirugía previa-, fueron sometidos a la colocación de la prótesis traqueal en T. En cinco de ellos el orificio de la traqueostomía estaba abierto al momento del diagnóstico de la FTA, mientras que en cuatro se requirió la reapertura del orificio de la traqueostomía previa. La prótesis permitió ocluir el orificio fistuloso facilitando la alimentación oral completa de los pacientes. Las sondas nasoentéricas o yeyunales fueron retiradas al momento de la colocación de la prótesis de Montgomery. 
Tabla I. Características de los pacientes con FTE.

\begin{tabular}{|c|c|}
\hline Pacientes (n) & 9 \\
\hline Edad (años) & $42.2 \pm 6.3$ \\
\hline Masculinos (\%) & 77.8 \\
\hline ARM (días) & $22.7 \pm 5.4$ \\
\hline Proteínas post-UTI (g \%) & $3.8 \pm 0.5$ \\
\hline Albúmina post-UTI (g \%) & $2.7 \pm 0.3$ \\
\hline Tiempo mejoría de las proteínas (meses) & $9.5 \pm 1.5$ \\
\hline \multicolumn{2}{|l|}{ Etiología de la fístula (\%) } \\
\hline CE & 1.1 \\
\hline TOT & 77.8 \\
\hline Estrangulamiento & I.I \\
\hline
\end{tabular}

La prótesis de Montgomery fue retirada en un promedio de $307 \pm 143$ días luego de la mejoría del estado nutricional

\section{Estado nutricional}

Los 9 pacientes sometidos a colocación de prótesis en $\mathrm{T}$ presentaron mal estado nutricional, las proteínas totales en sangre al momento de la consulta al servicio de broncoscopia fueron de $3,87 \pm 0.44 \mathrm{~g} / \mathrm{dl} v$ s. $6.56 \pm 0.44$ $\mathrm{g} / \mathrm{dl}$ al momento de decidir la cirugía $(\mathrm{p}<0.001)$, y la albúmina de $2.18 \pm 0.16 \mathrm{~g} / \mathrm{dl} v s .3 .4 \pm 0.16 \mathrm{~g} / \mathrm{dl}(\mathrm{p}<0.001)$ respectivamente (Figura 3). Tiempo requerido para mejoría de las proteínas fue de $9.5 \pm 1.5$ meses.

Durante el período de tratamiento con la prótesis de Montgomery en espera a la cirugía no se presentaron complicaciones asociadas a FTE y ningún paciente repitió infecciones respiratorias.

Una vez mejorado el estado nutricional de los pacientes se retiró la prótesis y se evaluó a través de una FBC la FTE. En la totalidad de los pacientes persistió la FTE por lo que se indicó el tratamiento quirúrgico correctivo. Solo en uno de los pacientes se realizó la seriada esofágica luego de la FBC para confirmar la persistencia de la comunicación por el pequeño tamaño del sitio de la fístula y la imposibilidad de poder evaluar la presencia de pasaje. Durante el seguimiento y en el perioperatorio no hubo fallecimiento de ningún paciente ni complicaciones posquirúrgicas y todos resolvieron satisfactoriamente la patología de base.

\section{DISCUSIÓN}

La FTE traumática posintubación se presenta en el $0.5 \%$ de los pacientes ventilados ${ }^{1}$. Esta baja prevalencia coincidió con las consultas por esta patología canalizadas en nuestro servicio.

La causa más común de FTE es posintubación por el sobre inflado de balón del tubo translaríngeo o de traqueostomía, generalmente por encima de los 30 $\mathrm{cmH}_{2} \mathrm{O}^{1,6,8}$, sumado al estado de shock, la traqueomala- cia y la intubación prolongada ${ }^{6,8}$. Se reporta una mortalidad de los pacientes con FTE y AVM del $0 \%$ al $28.6 \%{ }^{8}$. En los cinco pacientes diagnosticados en la TI ninguno falleció por esta causa una vez hecho el diagnóstico, y tampoco durante el período de estudio.

En los pacientes que reciben AVM en las TI es frecuente la desnutrición. Esto puede tener múltiples causas, aumento de los requerimientos metabólicos de los pacientes críticos bajo AVM, que no pudo ser suplido por las formas habituales de nutrición enteral y parenteral, la intolerancia, o las suspensiones no programadas de la alimentación ${ }^{4}$. La desnutrición constituye en sí mismo un factor pronóstico de mortalidad y complicaciones infecciosas bajo AVM y además un factor pronóstico para las complicaciones posquirúrgicas 4 .

En nuestra serie, los pacientes con FTE, al no ser considerados candidatos a cirugía al momento del diagnóstico por su inadecuado estado nutricional, el cierre de la brecha traqueoesofágica por la colocación de la prótesis en $\mathrm{T}$ de Montgomery permitió una adecuada alimentación que se expresó en la mejoría significativa de las proteínas totales/albúmina, en un promedio de $307 \pm 143$ días, y con la recuperación de su estado nutricional la cirugía fue exitosa. Además se pudo observar durante el seguimiento que con la prótesis traqueal no se evidenció recurrencia de broncoaspiración, por lo que no se requeriría una segunda prótesis a nivel esofágico.

El tratamiento definitivo de las fístulas es quirúrgico, como lo demuestra nuestra serie, en la que ningún paciente presentó el cierre del orificio fistuloso durante el tiempo de colocación de la prótesis.

La cirugía puede consistir en la separación de los órganos con o sin interposición de músculo, cierre simple de los orificios, derivación esofágica hasta la resección de laringe $y / o$ traqueal ${ }^{1,6,7}$.

Ninguno de los pacientes presentó complicaciones quirúrgicas precoces ni infecciosas posteriores hasta el año de seguimiento. No hubo mortalidad en nuestra serie en el estudio ni en el perioperatorio, como describe en la literatura de $2.6 \%$ con reoperación y sepsis ${ }^{5}$. La morbilidad relacionada con la necesidad de retraqueostomía, dehiscencia de sutura, recurrencia de la fístula, tampoco se presentó en nuestra serie 5 .

Dados los resultados favorables de nuestra serie, creemos que el manejo conservador inicial guiado por broncoscopia con la colocación de una prótesis en $\mathrm{T}$ Montgomery en un grupo seleccionado de pacientes es una alternativa válida y segura para conseguir la mejoría del estado nutricional como puente a la cirugía reparadora.

Para concluir, la FTE traumática es una patología poco prevalente. La morbimortalidad se disminuye drásticamente si se aleja la cirugía reparadora de la salida de la TI en pacientes con mal estado nutricional. El manejo endoscópico como puente hasta la cirugía, colocando una prótesis traqueal en T Montgomery que permi- 
ta la alimentación completa vía oral del paciente y realizar un seguimiento clínico-nutricional hasta la mejoría de las proteínas totales/albúmina, es una alternativa que en nuestro estudio mostró ser posible, segura y

\section{BIBLIOGRAFÍA}

I. Reed MF, Mathisen DJ.Tracheoesophageal fistula. Chest Surg Clin N Am 2003; I3:27|-89.

2. Payne DK, Anderson WM, Romero MD, Wissing DR, Fowler M. Tracheoesophageal fistula formation in intubated patients. Chest 1990:98:161-4.

3. Mooty RC, Rath P, Self M, et al. Review of Tracheo-Esophageal Fistula Associated with Endotracheal Intubation. I Surg 2007: 64:237-40

4. ZieglerTR. Parenteral Nutrition in the Critically III Patient. N Engl J Med 2009;36 |: 1088-97.

5. Muniappan A, Wain JC, Wright CD, et al. Surgical treatment of nonmalignant tracheoesophageal fistula: A thirty-five year experience. Ann Thorac Surg 20 I 3;95: I | 4 I-6. eficaz. La cirugía se realizó sin complicaciones una vez normalizados los valores en sangre de proteínas totales y albúmina.
6. Mathisen DJ, Grillo HC, Wain JC, Hilgenberg AD. Management of Acquired Nonmalignant Tracheoesophageal Fistula. Ann Thorac Surg 1991;52:759-65.

7. Shen RK,Allen MS, Cassivi SD, et al. Surgical managenent of acquired nonmalignant tracheoeophageal and bronchoesophageal fistulae. Ann Thorac Surg 2010;90:9 I4-9.

8. Marullia G, Loizzib M, Cardilloc G, et al. Early and late outcome after surgical treatment of acquired non-malignant tracheo-oesophageal fistulae. Eur J Cardio-Thorac Surg 20 I3;43;el 55-el 6l.

9. Braun T, Juenemann M, Viard M, et al. What is the value of fibreendoscopic evaluation of swallowing (FEES) in neurological patients? A cross-sectional hospital-based registry study BMJ Open 2018;8:e019016. 